\title{
Insilico studies of tau-protein kinase
}

Ranganadha R.A.

Department of Biotechnology, VFSTR University, Vadlamudi, Guntur 522213, Andhrapradesh, India

Corresponding Author Email: rangaaluri@gmail.com

https://doi.org/1018280/ama_b.610406

Received: 18 September 2018

Accepted: 26 November 2018

Keywords:

Alzheimer, tau protein, homology modeling, SWISS MODEL

\begin{abstract}
One of the most common types of dementia is Alzheimer's disease and it is related to tangles and plaques in the brain. Memory loss and symptoms like confusion and difficulty with remembering things indicate the onset of the disease. The symptoms are signs given by body to indicate brain cells death. In brain there are 100 billion nerve cells commonly known as neurons. Networks are formed when communication takes place between these nerve cells. Alzheimer's disease is very much similar to other forms of dementia as with time more and more brain cells die. Tau protein kinase enzyme catalyzes the transferring of the phosphate group to the oxygen side chains of the serine / threonine residues. Tau protein is one of the vital proteins that are present in the central nervous systems it helps to stabilize the neurons and microtubules. One of the main features of Alzheimer's is abundantly found neurofibrillary lesions that are made from Tau-protein that has been hyperphoshorylated. For stabilizing microtubules Tau-protein interact with tubulin. Auto assemblage of tangles with in the straight filaments and helical fragments takes place due to excessive phosphorylation of tau-protein. Dementia is caused to due malfunctioning of tau-protein that further causes Alzheimer's disease.
\end{abstract}

\section{INTRODUCTION}

Alzheimer's can be defined as neurodegenerative disease of the central nervous system. Alzheimer's disease is the most common form of dementia and is also known as Senile Dementia of the Alzheimer Type (SDAT) is mostly known as Alzheimer's. Alzheimer's was first elucidated by German psychiatrist and nueropathologist Alois Alzheimer in the year 1906; it is neurodegenerative, terminal disease that has no cure [1]. Alzheimer's is commonly seen in people who are of 65 years or above [2], even though it is not common Alzheimer's can occur at a young age. It was seen that 26.6 million people suffered from Alzheimer's worldwide according to the statistics recorded in 2006. By the year 2050 it is estimated that 107 million people will be suffering of Alzheimer's [3]. Alzheimer's is a unique disease but symptoms that are common can be seen in individuals. People often mistake the onset symptoms of Alzheimer's as old age problems or instance of stress [4]. In the starting stages it can be seen that the person loses memory and finds it difficult to remember even recent things. Behavior assessments and cognitive tests and brain scans are done by physicians or doctors if Alzheimer's is questionable. As the disease progresses further symptoms like irritation, moodiness, persistent confusion, hostility, loss of memory can be seen and slowly the sensibility of the patient is lost [5]. Little by little the functions of body comes to a halt and results in death of the patient. Because of the variations in the disease it is difficult to predict the progression in each person. Other than the main symptoms two other symptoms play an important role that can be related to the disease. Cognitive type of symptoms is the one of them and they include agnosia, aphasia, apraxia' both short and long term amnesia. One other type of symptom is of psychiatric type and they include depression, delusions hallucinations and changes in the personality. Alzheimer's can go undetected for years as it takes a lot of time to become properly illusive. Once the disease has been diagnosed the mean expectancy of the patient is seven years. It is seen that after diagnosing the Alzheimer's less than three percent of patients have lived for more than fourteen years [6]. The causes and the disease progression are still not really understood and this makes it a bit more difficult to assess the patient. According to research that has been done earlier it is said that the disease is caused due to plaques and tangles within the brain [7]. There is no proper treatment for the disease that can help to slow down the progress or prevent the disease from occurring and the treatments which are given have only minute effects. In order to identify a relevant treatment for Alzheimer's 500 clinical trials were conducted in the year 2008, but the results regarding the suitable drug still remains unknown [8]. In order to avoid Alzheimer's change in the habits within one's lifestyle is recommended but there is no evidence to prove that this will prevent or reduce the effects of Alzheimer's. In order to try and prevent the disease and to manage the disease balanced proper diet and adequate exercising and mental stimulations are suggested. It is important to take care of patients suffering from the disease as it is degenerative and is incurable. It is necessary that the patient receives proper care from spouse or a close relative. As the disease progresses the patients tend to become a burden to family and psychological, social, economic and physical elements of the caretaker's life are affected due to the patient. IN developed countries Alzheimer's disease it said to be one of the costliest diseases [9]. Tau-proteins are a type of proteins that are found in neurons and most commonly in central nervous system. Stabilization of microtubules is due to Tau proteins. A group of protein kinases regulate the Phosphorylation of tau, one of 
the examples for such kinases is $\mathrm{PKN}$ a serine/threonine kinase. Microtubule organization is disturbed when PKN is activated as it causes phosphorylation of PKN [10]. Self assembly of tangles in straight filaments and paired helical fragments is caused due to the hyperphosphorylation of the tau protein this is further linked to prognosis of Alzheimer's and other Tauopathies [11].

\section{METHODOLOGY}

A) The primary sequence of TAU PROTEIN KINASE (Acc.ID. Q5TCY1) was retrieved from Swiss Prot. BLAST software was used for homology search of TAU-PROTEIN KINASE

B) TAU-PROTEINKINASE crystal structure was modeled by using the SWISS MODEL server with (PDB ID: 4BTJ) as structural template.

C) Stereo-chemical quality of all the chains in a protein within the given PDB structure can be done by PROCHECK analysis. The regions that have unusual geometry are highlighted and the overall structural estimation is also provided.

\subsection{BLAST}

BLAST is a web based online tool which searches alignments that are of high scores between the target sequence and the homologous sequences that are present in the database by using Smith-Waterman algorithm and following a heuristic approach. BLASTP: In Blastp when a protein query is given the protein sequences that are most similar in the databases are given as output.

\subsection{SWISS MODEL}

SWISS MODEL is a fully automated protein structure comparative-modelling server, accessible via the ExPASY web server [12].

\subsection{PROCHECK}

In order to check the quality of the protein model it is important to evaluate the structural arrangement of protein from the atomic level, in order to do this PROCHECK was used [13].

\section{RESULTS AND DISCUSSION}

\subsection{Sequence retrieval and data collection}

By using UniProtKB/Swiss-Prot database the details of Tau protein kinase (Acc No. Q5TCY1) the information such as helix lengths, $\mathrm{N}$-and $\mathrm{C}$-terminus amino acid lengths etc., was retrieved. The sequence information is provided below.

Query sequence of Tau-tubulin kinase

>sp|Q5TCY1|TTBK1_HUMAN Tau-tubulin kinase 1 OS=Homo sapiens $\quad \mathrm{GN}=\mathrm{TTBK} 1 \quad \mathrm{PE}=1 \quad \mathrm{SV}=2$ WKVLKKIGGGGFGEIYEAMDLLTRENVALKVESAQQ PKQVLKMEVAVLKKLQGKDHVCRFIGCGRNEKFNYV VMQLQGRNLADLRRSQPRGTFTLSTTLRLGKQILESIE AIHSVGFLHRDIKPSNFAMGRLPSTYRKCYMLDFGLA RQYTNTTGDVRPPRNVAGFRGTVRYASVNAHKNREM
GRHDDLWSLFYMLVEFAVGQLPWRKIKDKEQVGMIK EKYEHRMLLKHMPSEFHLFLDHIASLDYFTKPDYQLI MSVFENSM

BLAST tool helps to search the regions of similarity between nucleotide or protein sequences by using local alignment algorithm. Statistical significance of the matches is calculated by comparing the protein or nucleotide query to the sequences in the database. By using the BLAST tool the target sequence i.e., Tau-protein kinase (UniProt ID: Q5TCY1) was searched against the sequences of protein in the database. By analyzing the BLAST results that are shown in Figure 1. it can be seen that maximum identity with target sequences are shown by three different proteins (PDB IDs: 4BTJ, 1CKJ, 2CK7). For further use out of these three sequences $4 \mathrm{BTJ}$ is selected (Figure 2).

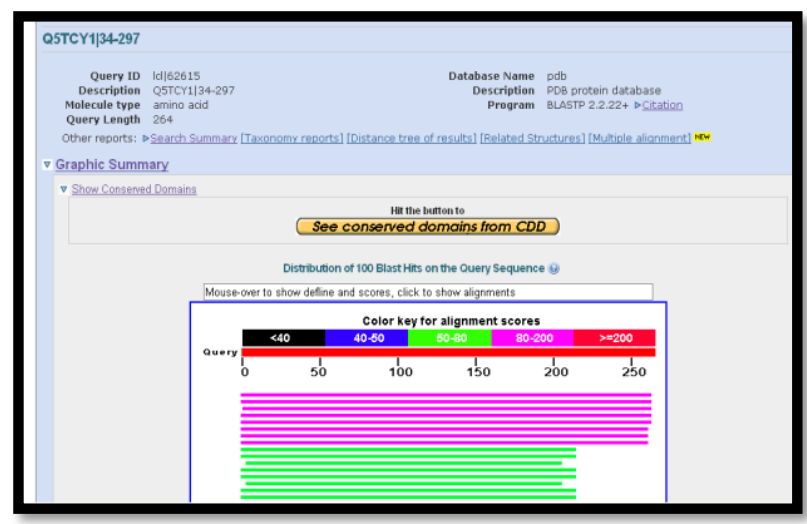

Figure 1. BLASTP result for Tau-protein kinase

\subsection{Three-dimensional structure prediction by SWISS MODEL}

4BTJ was retrieved through BLAST results and was taken as template, Q5TCY1 was taken as query sequence and Protein BLAST was performed, the 3D structure of Tauprotein kinase (Q5TCY1) was predicted by using the tool SWISS MODEL and can be seen in Figure 3. Taking 4BTJ_A chain as templates that are homologous to query sequence tau-protein kinase the 3D structure of the Tauprotein kinase (Q5TCY1) was predicted. A model with RMS deviation is 0.75 after superimposition of Tau-Protein kinase structure with templates 4BTJ was obtained.

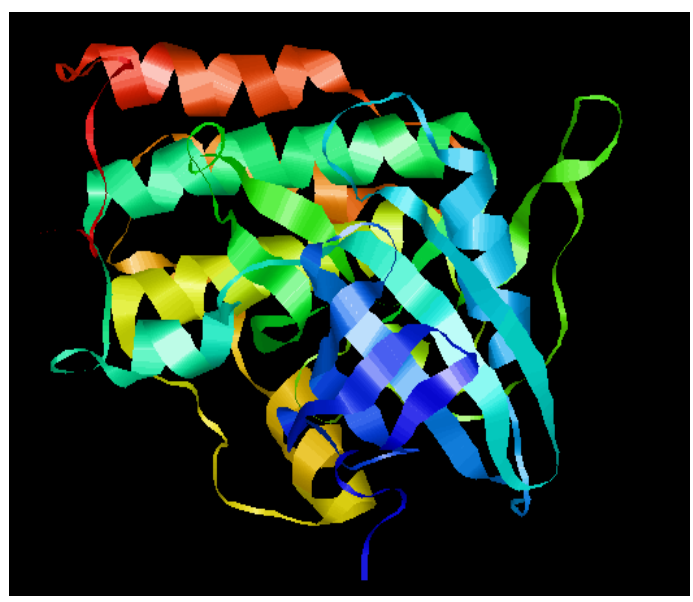

Figure 2. Crysyal structure of Tau-tubulin kinase 1 (PDB ID: 4BTJ) as structural template 


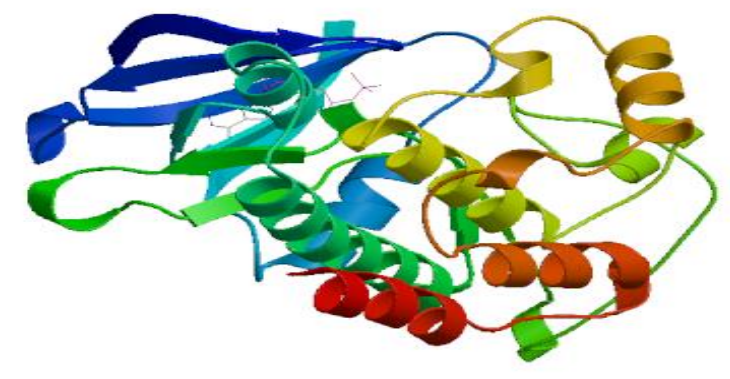

Figure 3. Homology model of Tau-protein kinase structure built by SWISS MODEL server

\subsection{Ramachandran plot}

The validation of the final model was carried out using Ramachandran plot computed with PROCHECK, program by checking the detailed residue-by-residue stereo-chemical quality of a protein structure.
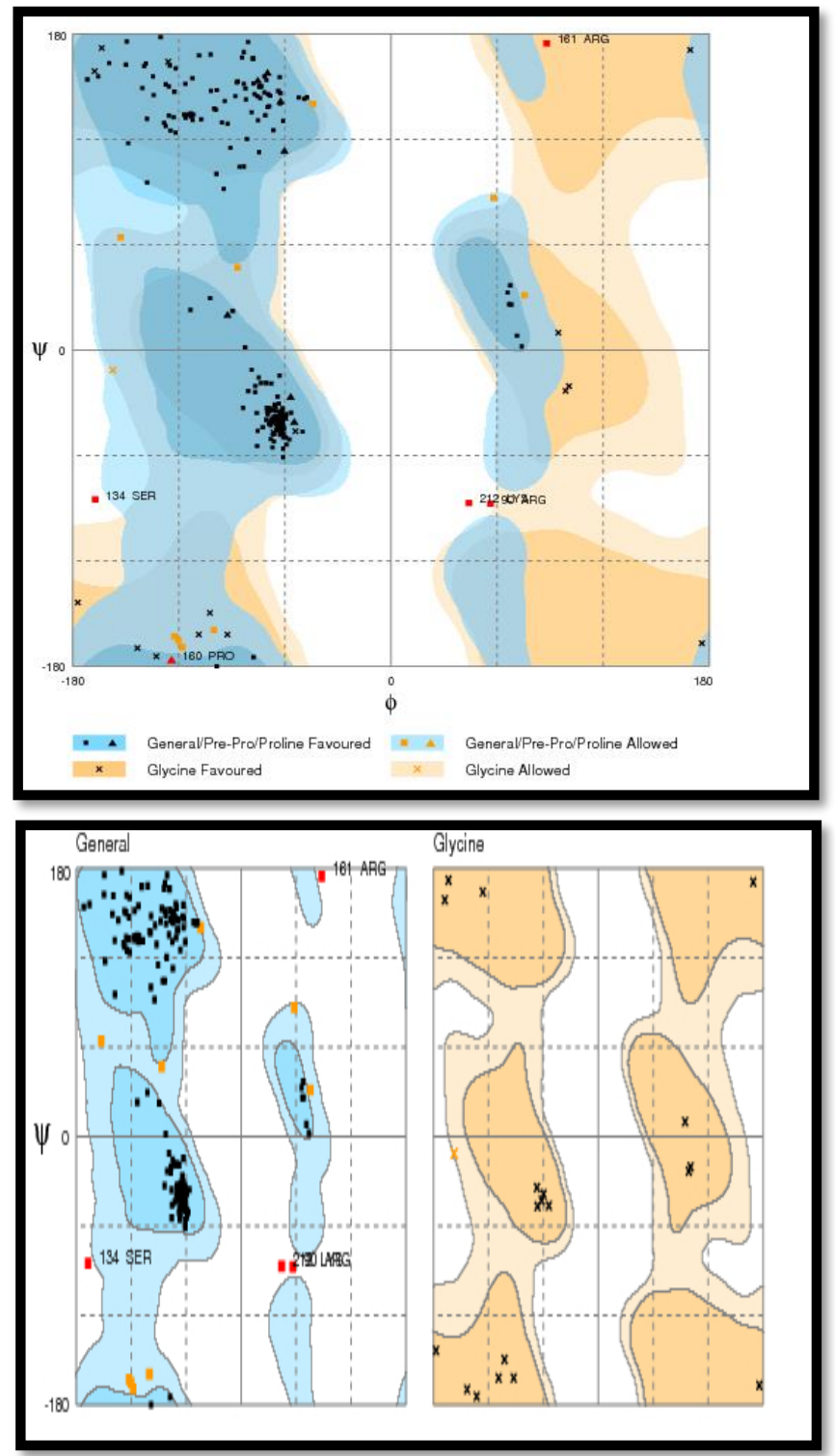

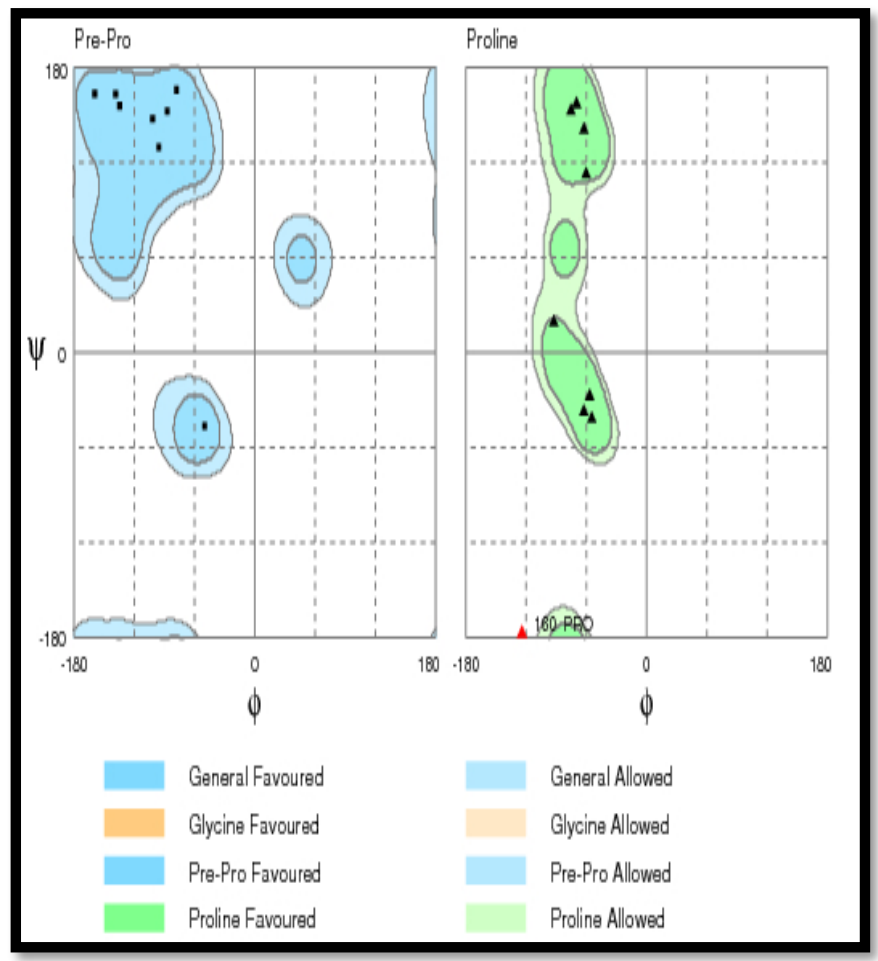

Figure 4. Ramachandran plot analysis of the generated model

The PROCHECK is used for stereochemical assessment of the model. Ramachandran plot was developed by Gopalasamudram Narayana Ramachandran and Viswanathan Sasisekharan. Iit is used to visualize dihedral angles $\Psi$ and $\Phi$ of amino acid residues in protein structure. It is commonly known as Ramachandran map or a Ramachandran diagram or a $[\Phi, \Psi]$ plot. It depicts the possible conformations of $\Phi$ and $\Psi$ angles for a polypeptide. Stereo chemical quality of a protein structure can be assessed by using Ramachandran plot. The model developed by PROCHECK was checked with the Ramachandran plot, Tau kinase protein had 247 $(94.3 \%)$ residues in the favored region against ( $98.0 \%$ expected), $10(3.8 \%)$ residues in allowed region against $(\sim 2.0 \%$ expected $)$ and 5 (1.9\% residues in outlier region) and it can be seen in the Figure 4.

\section{CONCLUSION}

In the current study we have modeled Tau protein kinase that plays a vital role in causing Alzheimer disease. By using BLASTP the template 4BTJ was found. SWISS MODEL was used for building the homology model of Tau protein kinase, by using PROCHECK the protein structure validation was done.

\section{REFERENCES}

[1] Berchtold NC, Cotman CW. (1998). Evolution in the conceptualization of dementia and Alzheimer's disease: Greco-Roman period to the 1960s. Neurobiology of Aging 19(3): 173-189. https://doi.org/10.1016/S01974580 (98)00052-9

[2] Brookmeyer R, Gray S, Kawas C. (1998). Projections of Alzheimer's disease in the United States and the public 
health impact of delaying disease onset. American Journal of Public Health 88(9): 1337-1342. https://doi.org/ 10.2105/AJPH.88.9.1337

[3] Brookmeyer R, Johnson E, Ziegler-Graham K, Arrighi HM. (2007). Forecasting the global burden of Alzheimer's disease. Alzheimer's and Dementia 3(3): 186-91. https://doi.org/10.1016/j.jalz.2007.04.381

[4] Waldemar G, Dubois B, Emre M. (2007). Recommendations for the diagnosis and management of Alzheimer's disease and other disorders associated with dementia: EFNS guideline. European Journal of Neurology 14(1): 1-26. https://doi.org/10.1111/j.14681331.2006.01605.x

[5] Tabert MH, Liu X, Doty RL, Serby M, Zamora D, Pelton GH, Marder K, Albers MW, Stern Y, Devanand DP. (2005). A 10-item smell identification scale related to risk for Alzheimer's disease. Annals of Neurology 58 (1): 155-160. https://doi.org/10.1002/ana.20533

[6] Mölsä PK, Marttila RJ, Rinne UK. (1986). Survival and cause of death in Alzheimer's disease and multi-infarct dementia. Acta Neurologica Scandinavica 74(2): 103107. 0404.1986.tb04634.x

[7] Mölsä PK, Marttila RJ, Rinne UK. (1995). Long-term survival and predictors of mortality in Alzheimer's disease and multi-infarct dementia. Acta Neurologica Scandinavica 91(3): 159-164. https://doi.org/10.1111/j.1600-0404.1995.tb00426.x

[8] Tiraboschi P, Hansen LA, Thal LJ, Corey-Bloom J. (2004). The importance of neuritic plaques and tangles to the development and evolution of AD. Neurology 62(11): 1984-1989. https://doi.org/10.1212/01.WNL.0000129697.01779.0A

[9] Murray J, Schneider J, Banerjee S, Mann A. (1999). EUROCARE: A cross-national study of co-resident spouse careers for people with Alzheimer's disease: II-A qualitative analysis of the experience of care giving. International Journal of Geriatric Psychiatry 14(8): 662667. https://doi.org/10.1002/(SICI)10991166(199908)14:8<662::AID-GPS993>3.0.CO;2-4

[10] Meek PD, McKeithan K, Schumock GT. (1998). Economic considerations in Alzheimer's disease. Pharmacotherapy 18(2): 68-73. https://doi.org/10.1002/j.1875-9114.1998.tb03880.x

[11] Taniguchi $T$, Kawamata $T$, Mukai $H$, Hasegawa $H$, Isagawa $\mathrm{T}$, Yasuda $\mathrm{M}$, Hashimoto $\mathrm{T}$, Terashima $\mathrm{A}$, Nakai M, Mori H, Ono Y, Tanaka C. (2001). Phosphorylation of tau is regulated by PKN. Journal of Biological Chemistry 276(13): 1002510031.https://doi.org/10.1074/jbc.M007427200

[12] Benkert P, Biasini M, Schwede T. (2011). Toward the estimation of the absolute quality of individual protein structure models. Bioinformatic 27(3): 343-350. https://doi.org/10.1093/bioinformatics/btq662

[13] Laskowski Roman A, Malcolm W, MacArthur, David SM, Jnaet MT. (1993) PROCHECK: A program to check the stereochemical quality of protein structures. Journal of Applied Crystallography 26(2): 283-291. https://doi.org/10.1107/S0021889892009944 